\title{
Crestal Bone loss around Anchor Units due to Banding -A Review
}

\author{
Dr. Ritika Chhalani ${ }^{1}$, Dr. Saravana Dinesh. S.P \\ ${ }^{\text {I}}$ Saveetha Dental College/ Saveetha University, India) \\ ${ }^{2}$ (Department of Orthodontics, Saveetha Dental College/ Saveetha University,India)
}

\begin{abstract}
Orthodontic fixed appliances are those that are fitted to the teeth for the purpose of aligning and correcting the inclination of teeth and cannot be removed by the patient at will. Various attachments are used in fixed appliance such as brackets and molar tubes that can be attached directly onto the teeth by a procedure known as bonding or can be welded to metal bands that are cemented onto the teeth by means of a procedure called banding. Banding has been used conventionally and is still in practice due to a variety of reasons. This article aims to bring about the problems faced with the age old practice of banding anchor teeth and also discusses why bonded options, though more prone to failures are still a better choice in many cases.
\end{abstract}

Keywords: Banding, Bonding, Bone loss

\section{Introduction}

Orthodontics involves the correction of malaligned teeth. According to the British Society for the study of Orthodontics; "Orthodontics includes the study of growth and development of the jaws and face particularly, and the body generally, as influencing the position of the teeth; the study of action and reaction of internal and external influences on the development, and the prevention and correction of arrested and perverted development ${ }^{[1]}$." In cases with proclined anterior teeth , correcting their inclination reqires them to be retracted with the help of an anchor, which is usually the molars. An anchor is a rigid point of support. Graber defined anchorage as, "The nature and degree of resistance to displacement offered by an anatomic unit for the purpose of effecting tooth movement. ${ }^{[2],}$ An anchor must be stable and must offer more resistance to the force that is required to move the other teeth. The resistance offered by a tooth is directly proportional to the surface area of its roots. Hence multi rooted teeth are more suited to serve as anchors. Anchorage can be of five types namely, simple, reciprocal, reinforced, inter maxillary and extra oral. Fixed appliances are those that are fitted to the teeth and cannot be removed by the patient at will. A variety of complex tooth movements are made possible with fixed appliances.

\section{Technique of banding anchor units}

Banding molars for anchorage is a common practice. Molar bands are usually made of 18-8 austenetic stainless steel. The outer surface is smooth and shiny to prevent plaque accumulation and to aid in easy maintenance. The inner surface is rough to enable retention of the cement. Molar bands can be pre-formed or custom made. Custom made bands are made from tapes of steel that are available in different dimensions, the molar tapes are usually wider and stiffer. Placement of the band requires mechanical separation of the teeth due to the presence of tight inter-proximal contacts. The Band is adapted to form a ring and is placed around the tooth, it is pinched and spot welded to suit the tooth dimensions, the excess material is cut off. The band is now adapted to fit the tooth contours and is spot welded once more. The gingival margins are trimmed and smoothened to avoid tissue injury. The attachments are fixed onto the band either by soldering or welding and then the band is cemented in place.

\section{Technique of bonding anchor units}

Fixing attachments directly over enamel using adhesive resins is called bonding. Acid etch technique developed by Buonocore in 1955 is used prior to bonding as it increases surface energy as well as surface area of enamel. 30-50\% orthophosrporic acid is applied over the surface for 15- 30 seconds. The etchant is rinsed away using water, the etched enamel has a frosty white appearance. High vacuum suction and cotton rolls are then used for adequate moisture control prior to bonding. The adhesive is placed over the attachment which is then appropriately positioned over the etched surface of the tooth and light cured.

\section{Banding Vs Bonding anchor teeth}

Banding is indicated in cases where buccal as well as lingual attachments are required, it is better suited to withstand heavy occlusal forces and is hence used in case of extra oral appliances like head gears. 
Bonded attachments are superior to their banded counterparts in that they enable better oral hygiene maintenance, and hence cause lesser damage to the tissues when compared to subgingivally placed bands that invariably accumulate plaque, the incidence of caries is therefore much lesser as well with bonded attachments. The need for antibiotic prophylaxis in patients who are at risk from bacteraemia is also eliminated. The major problem with bonded attachments has been frequent bond failure requiring multiple visits which has been claimed to hinder orthodontic treatment and for this sole reason banded attachments remain largely in vogue.

\section{The Periodontium}

The gingiva, periodontal ligament, alveolar bone and cementum function as a single unit and together constitute the periodontium. Gingiva is that part of the oral mucosa that covers the alveolar process of the jaws and surrounds the necks of the teeth. The periodontal ligament is a higly cellular and vascular connective tissue that suspends the tooth in its socket. It functions to transmit occlusal forces to the bone and acts as a shock absorber. Cells of the periodontal ligament participate in remodelling of cementum and bone. Cementum is the calcified, avascular mesenchymal tissue that forms the outer covering of the anatomic root. The alveolar process is that portion of the maxilla and mandible that forms and supports the tooth sockets. They are tooth dependent bony structures. Repositioning of teeth, accomplished in response to orthodontic procedures, rely on the adaptability of the alveolar bone and periodontal ligament. The alveolar bone is constantly remodelled and undergoes changes in height, width, contour and density ${ }^{[3]}$.

\section{Discussion}

Little research has been done on the postoperative changes seen on the periodontium following orthodontic therapy and much lesser to compare the postoperative periodontal changes seen in banded and bonded molars that take the brunt of the force as anchors. Bone, though rigid undergoes continous remodelling with cycles of resorption and formation. It is this property of bone that is put to use in orthodontics.

Orthodontic treatment aims at promoting oral health, however these treatments require high levels of oral hygiene maintenance. Fixed appliances harbour plaque and can be very damaging if the patient is not motivated towards maintenance of oral health. Majority of the patients require mechanotherapy at regular intervals as the duration of treatment is usually long. Gingivitis, pocket formation /alveolar bone loss or loss of attachment, root resorption, dental caries, enamel cracks and enamel decalcification are common complications associated with orthodontic therapy.

Loss of interproximal bone does occur irrespective of whether the teeth are bonded or banded but is more in banded molars as seen in Fig.1 and 2. This bone loss has been found to be more in Begg's technique when compared to pre-adjusted edge wise technique ${ }^{[4]}$. Bone loss was also more in the buccal aspect ${ }^{[5]}$. On application of an optimum orthodontic force, pressure and tension sites are created in the periodontal ligament on either side. Compression of the periodontal ligament on the pressure side leads to increase in vascularity and formation of osteoclasts, causing bone resorption. On the tension side, there is widening of the periodontal ligament which again leads to an increase in vascularity, however in this case, osteoblasts are formed causing bone deposition ${ }^{[6]}$. Periodontitis is progressive in nature and gingival inflammation during orthodontic tooth movement tends to augment resorption of the alveolar crest. Better oral hygiene maintenance retards bone loss in adults when compared to adolescents. Greater bone loss in adolescents has also been owed to more subgingival placement of molar bands due to lesser clinical crown height in adolescent patients ${ }^{[7]}$. However another study by Harris and Vaden ${ }^{[8]}$ claims that iatrogenic problems occur to a greater extent in adults as treatment involves repositioning of teeth in the alveolus whereas in adolescents the process is helped by the growth of the jaws and development of the alveolus.

Studies show that ill effects of orthodontic treatment may manifest as late as fifteen years posttreatment ${ }^{[7]}$. Tilting or intrusion of plaque infected teeth may cause a shift of supragingival plaque to a subgingival position, leading to bone and attachment loss ${ }^{[9]}$. Banded molars lose more attachment than bonded molars due to subgingival placement of molar bands. A study which compared periodontal changes in adolescents treated orthodontically by banded appliances and those treated with bonded appliances revealed that there was lesser plaque accumulation and hence comparatively lesser gingival inflammation around bonded teeth $^{[10]}$ as seen in Fig. 3 and 4. According to a study comparing the periodontal status of bonded and banded molars, the banded molars exhibit more signs of gingivitis and more attachment loss, both during and after treatment. Among the molars that were banded in the study, the maxillary molars showed more evidence of loss of attachment. This change observed in banded molars can be due to the fact that plaque accumulation is more in overhanging gingival margins of orthodontic bands as these areas are difficult to clean. Another explanation that was given is the mechanical injury caused by subgingival placement of orthodontic bands ${ }^{7}$.

\section{Figures}




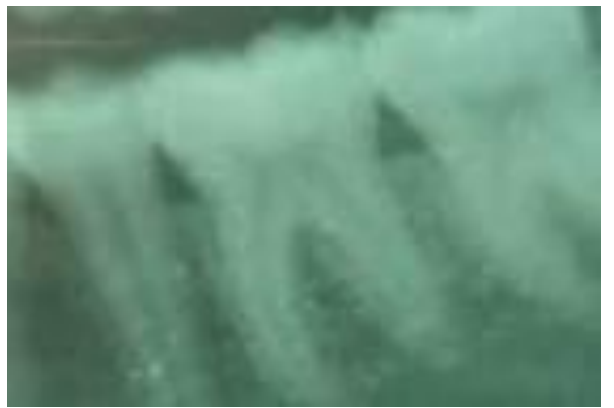

Figure 1: Pre- operative radiograph

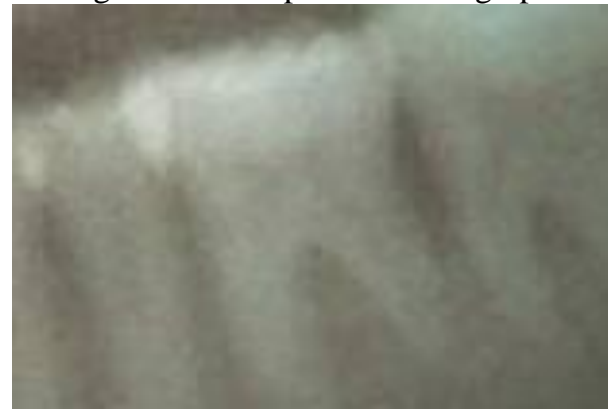

Figure 2: Post- operative radiograph of banded molar

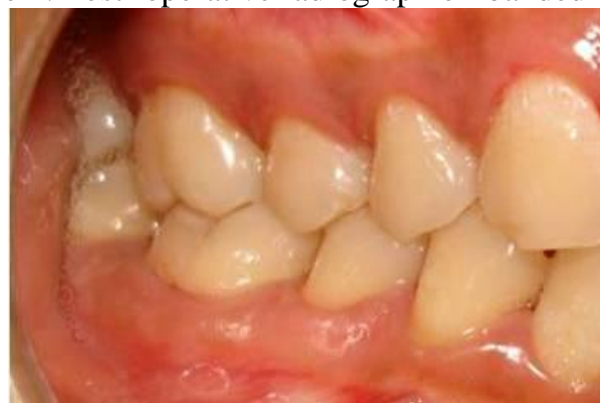

Figure 3: Pre- operative

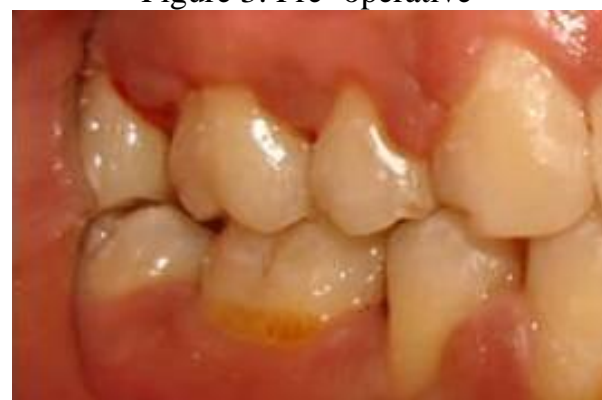

Figure 4: Post operative gingivitis in banded molar

\section{Conclusion}

It has been proved beyond doubt that bonded molar attachments are kinder to the periodontium than banded ones, however bond failures due to poor moisture control as well as due to occlusal stresses has lead to banded attachments still being preferred by many operators. The question that arises here is, is it acceptable to treat one condition and cause another? When do the risks outweigh the benefits? This decision must be made with full knowledge of what the ill effects could be.

\section{References}

[1] British society for the study of Orthodontics; 1922

[2] Graber TM: Orthodontics: Principles and Practice. WB Saunders , 1998

[3] Carranza's clinical Periodontology by Newman, Takei and, Klokkevold and Carranza; $10^{\text {th }}$ edition,;2006.

[4] "Evaluation of proximal alveolar bone level changes during orthodontic treatment-a comparative clinical study." The Journal of Indian Orthodontic Society: 48.

[5] Zachrisson, bjørn u., and Lars alnæs. "Periodontal condition in orthodontically treated and untreated individuals II. Alveolar bone loss: radiographic findings." The Angle Orthodontist 44.1 (1974): 48-55. 
[6] Orthodontics, The Art and Science by S.I. Bhalajhi; $5^{\text {th }}$ edition pg 395

[7] Boyd, Robert L., and Sheldon Baumrind. "Periodontal considerations in the use of bonds or bands on molars in adolescents and adults." The Angle Orthodontist 62.2 (1992): 117-126.

[8] Dyer, Gregory S., Edward F. Harris, and James L. Vaden. "Age effects on orthodontic treatment: adolescents contrasted with adults." American Journal of Orthodontics and Dentofacial Orthopedics 100.6 (1991): 523-530.

[9] Nelson, Paul A. "Alveolar bone loss of maxillary anterior teeth in adult orthodontic patients." American journal of orthodontics and dentofacial orthopedics 111.3 (1997): 328-334.

[10] Zachrisson, Bjørn U. "A post treatment evaluation of direct bonding in orthodontics." American journal of orthodontics 71.2 (1977): 173-189. 